\title{
Awareness and Knowledge of Smoking-Related Cancers Among University Students in Jordan
}

\author{
Ayoub A. Innabi , Dina A. Ammari, Wa'el J. K. Tuqan \\ Faculty of Medicine, University of Jordan, Amman, Jordan \\ *Corresponding author: ayoubinnabi@yahoo.com
}

Received May 27, 2014; Revised June 07, 2014; Accepted June 11, 2014

\begin{abstract}
Background: Jordan, a Middle Eastern country, has a high prevalence rate of smoking. However, little research was done to evaluate knowledge of smoking-related cancers in the region. Objective: To assess knowledge of smoking-related cancers among Jordanian university students. Design: Cross-sectional study conducted in July, 2013, using a self-administered online questionnaire. The questionnaire was sent to students from University of Jordan. Results: The final sample consisted of 230 students. The majority of participants (98.7\%) were aware that smoking has harmful health effects and (95.7\%) agreed that smoking causes cancer. Most of the participants agreed that smoking causes lung cancer (97.8\%), oral cancer (83.0\%), laryngeal cancer (82.6\%), pharyngeal cancer (80.0\%), and esophageal cancer (63.0\%), while less than half of the participant thought that smoking causes AML (46.1\%), stomach cancer (36.1\%), kidney cancer (32.6\%), cervical cancer (19.6\%), pancreatic cancer (19.1\%), and bladder cancer (14.8\%). Females showed more knowledge about smoking-related cancers than males $(\mathrm{p}<0.05)$ but the there was no significant difference between smokers and non-smokers. Conclusion: These findings show that most of the students know that lung cancer is caused by smoking. However, there is a lower knowledge of other smoking-related cancers. Our study suggests that more efforts should be done to increase the awareness of the adverse health hazards of smoking especially that are related to cancer.
\end{abstract}

Keywords: smoking, awareness, knowledge, smoking-related cancers, university students, Jordan

Cite This Article: Ayoub A. Innabi, Dina A. Ammari, and Wa'el J. K. Tuqan, "Awareness and Knowledge of Smoking-Related Cancers Among University Students in Jordan.” American Journal of Cancer Prevention, vol. 2, no. 2 (2014): 20-23. doi: 10.12691/ajcp-2-2-1.

\section{Introduction}

Cigarette smoking is considered to be the single most important factor responsible for the high prevalence of cancer and for death related to cancer [1]. At least, 4000 chemical compounds are found in a cigarette, of which 60 are known carcinogens [2]. Ninety percent of lung cancer cases have been directly attributed to cigarette smoking. Cigarette smoking also been considered to be a cause of laryngeal, oropharyngeal, and hypopharyngeal cancers. In addition, it has been shown to increase the risk of leukemia, sinonasal cancer, nasopharyngeal cancer, and esophageal cancer. Smoking was also established as a risk factor for stomach cancer, pancreatic cancer, transitional cell carcinoma of the bladder, ureter, and renal pelvis, as well as the cancers of the uterine cervix and kidney [1,3].

Smoking cigarettes is expected to be one of the causes of an anticipated $181 \%$ increase in cancer mortality in the Middle East by the year 2020, in comparison to a $26 \%$ expected increase in the developed countries [4]. Jordan, a Middle Eastern country, has high prevalence rates of smoking as it ranked the fourth highest Arab country in smoking rates [5]. Some reports showed that smoking among the adult population in Jordan is estimated to be around $51 \%$ for males and $8 \%$ for females, which is unacceptably high especially for males [6]. A study conducted in 2002 showed that $50.2 \%$ of university student males and 6.5\% among females are smokers [7]. More recent studies reported smoking rates of 33\% [8] and 29\% [9] among Jordanian university students. Thus smoking is a serious public health problem in Jordan.

The aim of this study is to assess the knowledge of smoking-related cancers among university students in Jordan. Those educated students are important targets for behavioral changes as models for others in their environment and as potential society decision makers in the future [10].

\section{Methodology}

This is a cross-sectional study that was conducted in July, 2013 among students attending University of Jordan that is situated in Amman, the capital city of Jordan.

An online self-administered questionnaire was sent randomly to 500 university students. Participants were recruited from University of Jordan pages on the social network website Facebook (www.facebook.com). Facebook was selected because it is the most popular social networking website used among university students $[11,12]$. The link of the questionnaire was sent through 
private messages to the students with a detailed explanation about the study.

The questionnaire was developed in Arabic. It was piloted on a group of university students to ensure clarity of the questions. Minor alterations in wording some questions were made. Data collected during the pilot study were not included in the study. The average time need to complete the questionnaire was around five minutes.

The questionnaire comprised three sections. The first section included basic socio-demographics variables such as age, gender, faculty, and smoking status.

The second section assessed awareness and knowledge regarding harmful effects of smoking on health. Questions used to assess awareness of the participants asked whether smoking has harmful effects on health, and whether it causes cancer. The cancer types selected in the questionnaire were based on a list from the Centers for Disease Control and Prevention official website (CDC, 2013). Questions used to assess knowledge of participants regarding specific cancers asked separately whether smoking was a cause of the following cancers or not: Blood cancer like acute myelogenous leukemia (AML), bladder cancer, cervical cancer, esophageal cancer, kidney cancer, lung cancer, oral cancer, pharyngeal cancer, laryngeal cancer, pancreatic cancer, and gastric cancer. Response categories were: "Yes”, “No”, “Don't know”. Each correct answer for the smoking-related cancers was given 1 point. A summation for the 11 items gives a total score of 11. A possible minimum score is 0 and a maximum score is 11. A higher score indicates a greater knowledge.

The third section comprised two questions: Do you believe that you have enough knowledge about smokingrelated cancers? Do you want to know more about smoking-related cancers? Response categories to the questions in this section were: "Yes”, "No", "Don't know".

Statistical Package for Social Sciences (SPSS) version 19.0 was used for statistical analysis. Simple frequencies and percentages were used to express qualitative (categorical) variables. Chi-square test was used to compare frequencies when applicable. For quantitative variables, means and standard deviations were used. T-test was used to compare knowledge scores among groups. A p-value of $<0.05$ was considered significant.

\section{Results}

A total of 247 university students responded to the questionnaire. Seventeen students from health and medical faculties (Medicine, Nursing, and Dentistry faculties) were excluded as we thought they may affect the results due to their medical background. A final total of 230 were included and had their results compared.

Table 1. General Characteristic of the Participants

\begin{tabular}{|c|c|c|}
\hline Characteristic & Male, n (\%) & $90(39.1)$ \\
\hline \multirow{2}{*}{ Gender } & Female, n (\%) & $140(60.9)$ \\
\cline { 2 - 3 } & \multicolumn{2}{|c|}{$20.7(1.6)$} \\
\hline Age, mean (SD) & Current smoker, n (\%) & $35(15.2)$ \\
\hline \multirow{2}{*}{ Smoking status } & Non-smoker, n (\%) & $195(84.8)$ \\
\cline { 2 - 3 } &
\end{tabular}

General characteristics of the participants are described in Table 1. Mean age of the participants was 20.7 (SD: 1.6). More than half of the participants were females
(60.9\%). Most of the participants were non-smokers (84.8\%).

The majority of participants (98.7\%) were aware that smoking has harmful effects on general health and 95.7\% agreed that smoking causes cancer.

Regarding knowledge of cancers caused by smoking, most of the participants agreed that smoking causes lung cancer (97.8\%), oral cancers (83.0\%), laryngeal cancers (82.6\%), pharyngeal cancers (80.0\%), and esophageal cancer $(63.0 \%)$. While less than half of the participants agreed that smoking causes AML (46.1\%), stomach cancer $(36.1 \%)$, kidney cancers (32.6\%), cervical cancer (19.6\%), pancreatic cancer (19.1\%), and bladder cancer (14.8\%). Knowledge of smoking-related cancers according to age and gender are illustrated in Table 2.

Table 2. Percentage of Correct Answers for each Smoking-Related Cancer According to Smoking Status and Gender

\begin{tabular}{|c|c|c|c|c|c|c|c|}
\hline \multirow{2}{*}{ Cancer } & \multicolumn{3}{|c|}{ Smoking Status (\%) } & \multicolumn{3}{c|}{ Gender (\%) } & $\begin{array}{c}\text { Tota } \\
\text { 1 } \\
(\%)\end{array}$ \\
\cline { 2 - 9 } & $\begin{array}{c}\text { Smok } \\
\text { er }\end{array}$ & $\begin{array}{c}\text { non- } \\
\text { smok } \\
\text { er }\end{array}$ & $\begin{array}{c}\text { p - } \\
\text { value } \\
*\end{array}$ & $\begin{array}{c}\text { Mal } \\
\text { e }\end{array}$ & $\begin{array}{c}\text { femal } \\
\text { e }\end{array}$ & $\begin{array}{c}\text { p - } \\
\text { value } \\
*\end{array}$ & \\
\hline Bladder & 11.4 & 15.4 & 0.06 & 13.3 & 15.7 & 0.73 & 14.8 \\
\hline Pancreas & 11.4 & 20.5 & $<.05$ & 15.6 & 21.4 & $<.05$ & 19.1 \\
\hline Cervix & 14.3 & 20.5 & 0.67 & 16.7 & 21.4 & 0.47 & 19.6 \\
\hline Kidney & 17.1 & 35.4 & $<.05$ & 27.8 & 35.7 & 0.26 & 32.6 \\
\hline Stomach & 31.4 & 36.9 & 0.06 & 28.9 & 40.7 & $<.05$ & 36.1 \\
\hline AML & 45.7 & 46.2 & $<.05$ & 48.9 & 44.3 & $<.05$ & 46.1 \\
\hline $\begin{array}{c}\text { Esophag } \\
\text { uns }\end{array}$ & 48.6 & 65.6 & $<.05$ & 52.2 & 70.0 & $<.05$ & 63.0 \\
\hline Pharynx & 77.1 & 80.5 & $<.05$ & 71.1 & 85.7 & $<.05$ & 80.0 \\
\hline Larynx & 80.0 & 83.1 & 0.40 & 77.8 & 85.7 & 0.23 & 82.6 \\
\hline Oral & 82.9 & 83.1 & $<.05$ & 76.7 & 87.1 & 0.12 & 83.0 \\
\hline Lung & 94.3 & 98.5 & $<.05$ & 95.6 & 99.3 & 0.13 & 97.8 \\
\hline
\end{tabular}

*The two groups were compared using Chi-square test.

On the knowledge score that was calculated for each participant to assess their knowledge, females had a higher score than males $(6.07$ versus $5.25, \mathrm{p}<0.05)$. Although non smokers had a higher score than smokers, it was not statistically significant (5.14 versus $5.86, \mathrm{p}>0.05)$. Table 3.

Table 3. Comparison between the Mean Scores of Knowledge between Males and Females, and between Smokers and NonSmokers

\begin{tabular}{|c|c|c|c|}
\hline Variable & Category & Mean (SD) & \multirow{2}{*}{ p-value* } \\
\hline \multirow{2}{*}{ Gender } & Male & $5.24(2.75)$ & \multirow{2}{*}{$<.05$} \\
\cline { 2 - 3 } & Female & $6.07(2.32)$ & \multirow{2}{*}{0.12} \\
\hline \multirow{2}{*}{ Smoker } & Current smoker & $5.14(2.63)$ & \\
\cline { 2 - 3 } & Non-smoker & $5.86(2.50)$ & \\
\hline
\end{tabular}

*The two groups were compared using t-test.

Less than half of the participants (33.5\%) felt that they had enough knowledge about smoking-related cancers. Most of the participants (78.7\%) wanted to know more about smoking-related cancers.

\section{Discussion}

To our knowledge, this is the first study in the region to investigate the knowledge of university students about smoking-related cancers. Few studies that were conducted in Jordan discussed the knowledge of university students about smoking health risks and lung cancer was the only cancer that was studied $[7,13]$.

The majority of participants (98.7\%) recognized that smoking has adverse effects on health and that it is causes 
to cancer (95.7\%), but a smaller proportion recognized the actual types of cancer. A recent study found that $84 \%$ of Pakistani women reported that smoking has a harmful effect on their health and only $14 \%$ of them agreed that smoking can cause cancer [14]. Another study from northern Sudan reported that $96 \%$ of the participants agreed that smoking can cause cancer [15].

Our study found that knowledge of smoking-related cancers was high with regard to lung cancer (97.8\%), but lower for other cancers. Most of the participants agreed that smoking is a cause of lung cancer (97.8\%) which is higher than that found among Malaysian university students (87.4\%) [16]. In another study from Malaysia, $91.3 \%$ of the study participants mentioned that cigarette smoking is a risk factor for lung cancer [17]. In another study from china, $77.5 \%$ of the participants knew that smoking causes lung cancer [18]. This might be attributed to the important role of pictorial warnings about lung cancer on cigarette packages in Jordan [19] and due to the role of media and health education campaign in emphasizing the adverse effects of smoking on the lungs.

More than half of the participants recognized the association between aerodigestive tract cancers and smoking. The rate of awareness among university students concerning smoking and oral cancer was 83\%. A higher rate was found among undergraduate medical and nursing students in a private university in Malaysia (95.5\%) [20], whereas lower rates of awareness of head and neck cancers were found among adult American smokers (67.8\%) [21], and among adults in southern Iran (73.6\%) [22].

Less than half of the participants recognized smoking as a risk for blood, stomach, kidney, cervical, pancreatic, and bladder cancers. These results indicate that the depth of knowledge on smoking health hazards is inadequate among university students. The poor knowledge about the association between smoking and these types of cancer is consistent with other studies [21,23,24,25,26]. For example, a study conducted in 2002 among Asian Americans found that participants awareness of the association between bladder, pancreatic, cervical and kidney cancers and smoking ranged between $49.2 \%$ and $56.8 \%$ [25]. Another US study found that 36\% of patients visiting the urology clinic recognized smoking as a risk factor for bladder cancer compared to only $14.8 \%$ in our study [23]. Taking cervical cancer as another example, one study found that $31.8 \%$ of Indian female university students agreed that smoking can cause cervical cancer compared to $19.6 \%$ in our study [26]

Most prior studies have found that females had higher knowledge scores than males regarding various health hazards of smoking [21,25]. In our study, females were found to have significantly higher scores than males regarding the association between smoking and various types of cancer. This is consistent with another study conducted in Jordan, where female university students were more aware of the harmful effects of smoking than males [7].

Contrary to other studies, there was no significant difference in knowledge scores between current smokers and current non-smokers [7,18,27,28]. A prior study among university students in Jordan, with $28.6 \%$ of their sample being current smokers, found that non-smokers were more aware of the harmful effects of smoking [7].
This discrepancy between the results may be due to the small number of smokers in our study, so further studies with larger sample are recommended.

An interesting point is that most of the participants felt that they do not have enough knowledge about smokingrelated cancers and most of the participants (78.7\%) reported willingness to know more about smoking-related cancers. This might be a good indicator that programs directed toward increasing awareness about health hazards of smoking will be effective among university students.

These findings suggest that there is a need for public education about less known harmful effects of smoking in order to help lower both smoking prevalence and smoking-related cancers. Studies have demonstrated that increased knowledge about tobacco smoking is a preventive factor for initiating smoking, and results in lower rates of tobacco consumption among population $[14,29]$. Targeting health behaviors of university students can be of great value, as college years can be viewed as a "window of opportunity" for preventing initiation or to encourage early quitting [10]. Thus, a higher level of awareness among university students should be achieved by anti-smoking programs using various modalities including anti-smoking posters, campaigns and lectures.

At the national level, warning labels on cigarette packages are an important source for information about the health risks of smoking and the different types of cancer related to smoking. Research has shown that smokers living in societies where these labels are mandated by the government reported higher health knowledge [30]. Thus their use is recommended to increase awareness of smoking-related cancers. In addition, mass media campaigns directed against smoking can decrease the prevalence of smoking by reducing rates of smoking initiation in young people as well as increasing the rates of quitting [31,32]. Therefore, more efforts should be taken through mass media, including TV, radio, newspapers, as well as social networking websites such as Facebook to provide information about the danger of tobacco smoking especially in relation to cancer. Health care professionals have a similar responsibility to increase the awareness regarding various health risks associated with smoking including different types of cancer.

In summary, our study extends the literature by investigating university students' knowledge about smoking-related cancers. Although knowledge among participants that lung cancer can be caused by smoking was high, knowledge about other cancers caused by smoking was lower. Our data suggest that public need to be better informed about smoking-related cancers other than lung cancer.

\section{Acknowledgments}

The authors would like to thank all the students who participated in this study.

\section{References}

[1] Lee, H., S.J. Yoon, and H.S. Ahn, Measuring the burden of major cancers due to smoking in Korea. Cancer Sci, 2006. 97 (6): p. 530-4. 
[2] Das, S.K., Harmful health effects of cigarette smoking. Mol Cell Biochem, 2003. 253 (1-2): p. 159-65.

[3] Levitz, J.S., T.P. Bradley, and A.L. Golden, Overview of smoking and all cancers. Med Clin North Am, 2004. 88(6): p. 1655-75, xiii.

[4] Rastogi, T., A. Hildesheim, and R. Sinha, Opportunities for cancer epidemiology in developing countries. Nat Rev Cancer, 2004. 4 (11): p. 909-17.

[5] Shishani, K., et al., Smoking patterns among Jordanian health professionals: a study about the impediments to tobacco control in Jordan. Eur J Cardiovasc Nurs, 2011. 10 (4): p. 221-7.

[6] Merrill, R.M., et al., Nurse and physician patient counseling about tobacco smoking in Jordan. Promot Educ, 2008. 15 (3): p. 9-14.

[7] Haddad, L.G. and M.Z. Malak, Smoking habits and attitudes towards smoking among university students in Jordan. Int J Nurs Stud, 2002. 39 (8): p. 793-802.

[8] Naddaf, A., The social factors implicated in cigarette smoking in a Jordanian community. Pak J Biol Sci, 2007. 10 (5): p. 741-4.

[9] Khabour, O.F., et al., Waterpipe tobacco and cigarette smoking among university students in Jordan. Int J Tuberc Lung Dis, 2012. 16 (7): p. 986-92.

[10] Thomas, J.L., et al., Abstinence and relapse rates following a college campus-based quit \& win contest. J Am Coll Health, 2010. 58 (4): p. 365-72.

[11] Brockman, L.N., et al., Hookah's new popularity among US college students: a pilot study of the characteristics of hookah smokers and their Facebook displays. BMJ Open, 2012. 2 (6).

[12] Egan, K.G. and M.A. Moreno, Alcohol references on undergraduate males' Facebook profiles. Am J Mens Health, 2011. 5 (5): p. 413-20.

[13] Kofahi, M.M. and L.G. Haddad, Perceptions of lung cancer and smoking among college students in Jordan. J Transcult Nurs, 2005. 16 (3): p. 245-54.

[14] Bhanji, S., et al., Factors related to knowledge and perception of women about smoking: a cross sectional study from a developing country. BMC Womens Health, 2011. 11: p. 16.

[15] Ahmed, H.G., Survey on knowledge and attitudes related to the relation between tobacco, alcohol abuse and cancer in the northern state of Sudan. Asian Pac J Cancer Prev, 2013. 14 (4): p. 2483-6.

[16] Al-Naggar, R.A., et al., Prevalence and of smoking and associated factors among Malaysian University students. Asian Pac J Cancer Prev, 2011. 12 (3): p. 619-24.

[17] Al-Naggar, R.A. and S.Y. Kadir, Lung cancer knowledge among secondary school male teachers in Kudat, Sabah, Malaysia. Asian Pac J Cancer Prev, 2013. 14 (1): p. 103-9.
[18] Yang, Y., et al., Awareness of tobacco-related health hazards among adults in China. Biomed Environ Sci, 2010. 23 (6): p. 43744.

[19] Hawari, F.I., et al., Perceptions of young Jordanian adults to proposed anti-tobacco pictorial warning labels. BMC Public Health, 2011. 11: p. 414.

[20] Al Dubai, S.A., et al., Awareness and knowledge of oral cancer among university students in Malaysia. Asian Pac J Cancer Prev, 2012. 13 (1): p. 165-8.

[21] Oncken, C., et al., Knowledge and perceived risk of smokingrelated conditions: a survey of cigarette smokers. Prev Med, 2005. 40 (6): p. 779-84.

[22] Tadbir, A.A., et al., Evaluation of levels of knowledge about etiology and symptoms of oral cancer in southern Iran. Asian Pac J Cancer Prev, 2013. 14 (4): p. 2217-20.

[23] Nieder, A.M., et al., Are patients aware of the association between smoking and bladder cancer? J Urol, 2006. 176 (6 Pt 1): p. 2405-8; discussion 2408.

[24] Anastasiou, I., et al., Patient awareness of smoking as a risk factor for bladder cancer. Int Urol Nephrol, 2010. 42 (2): p. 309-14.

[25] Ma, G.X., et al., Perceived risks of certain types of cancer and heart disease among Asian American smokers and non-smokers. J Community Health, 2002. 27 (4): p. 233-46.

[26] Joy, T., et al., Awareness of cervix cancer risk factors in educated youth: a cross-sectional, questionnaire based survey in India, Nepal, and Sri Lanka. Asian Pac J Cancer Prev, 2011. 12 (7): p. 1707-12.

[27] Murphy-Hoefer, R., S. Alder, and C. Higbee, Perceptions about cigarette smoking and risks among college students. Nicotine Tob Res, 2004. 6 Suppl 3: p. S371-4.

[28] Minh An, D.T., et al., Knowledge of the health consequences of tobacco smoking: a cross-sectional survey of Vietnamese adults. Glob Health Action, 2013. 6: p. 1-9.

[29] Xu, Y., et al., Tobacco knowledge among adults in Zhejiang Province, China. PLoS One, 2013. 8 (3): p. e59172.

[30] Hammond, D., et al., Effectiveness of cigarette warning labels in informing smokers about the risks of smoking: findings from the International Tobacco Control (ITC) Four Country Survey. Tob Control, 2006. 15 Suppl 3: p. iii19-25.

[31] Hafstad, A., et al., Provocative appeals in anti-smoking mass media campaigns targeting adolescents--the accumulated effect of multiple exposures. Health Educ Res, 1997. 12 (2): p. 227-36.

[32] Flynn, B.S., et al., Mass media and school interventions for cigarette smoking prevention: effects 2 years after completion. Am J Public Health, 1994. 84 (7): p. 1148-50. 